\title{
BACTERIOLOGICAL PROFILE AND ANTIBIOTIC SUSCEPTIBILITY PATTERN OF BLOOD CULTURE ISOLATES FROM BURN PATIENTS
}

\author{
Rajpal Kamlesh', Kumar Ajay², Nag Sanjay33, Lal Arjun ${ }^{4}$, Mahto S. P 5 \\ ${ }^{1}$ Assistant professor, Department of Microbiology, ANMMCH, Gaya. \\ ${ }^{2}$ Senior Resident, Department of Microbiology, IGIMS, Patna. \\ ${ }^{3}$ Assistant Professor, Department of Microbiology, ANMMCH, Gaya. \\ ${ }^{4}$ Associate Professor, Department of Microbiology, ANMMCH, Gaya. \\ 5Professor, Department of Microbiology, ANMMCH, Gaya.
}

ABSTRACT

\section{BACKGROUND}

Infection in a burn patient is a leading cause of morbidity and mortality and remains one of the most challenging concerns for doctors.

The aim of this study is to determine the bacteriological profile of blood stream infections and their antibiotic sensitivity pattern.

\section{MATERIALS AND METHODS}

One hundred sixteen blood samples were collected from acutely burnt patients, being treated in the Rajendra Surgical Ward of the Patna Medical College and Hospital, Patna. This descriptive study was conducted from July 2004 to August 2005.

\section{RESULTS}

In the present series of study, it is observed that out of a total of 17 isolates (5.84\%) of Staphylococcus aureus, 14 isolates (i.e. 82.35\%) were sensitive to Cloxacillin. Antibiotics resistant to Staphylococcus aureus were Gentamicin, Amikacin, Cotrimoxazole, Lomefloxacin, out of a total of 27 (i.e. 9.28\%) isolates of Pseudomonas aeruginosa, 11 isolates (i.e. 40.74\%) were sensitive to Gentamicin and 13 isolates (i.e. 48.15\%) were sensitive to Imipenem. Isolates were resistant to Cloxacillin, Ampicillin, Cefazolin and Cotrimoxazole. Out of a total of 7 isolates (i.e. $2.41 \%$ ) of Proteus spp., 6 isolates (i.e. 85.71\%) were sensitive to Imipenem. Isolates were resistant to Cloxacillin, Cefazolin, Cefoperazone. Out of a total of 6 isolates (i.e. $2.06 \%$ ) of Klebsiella spp., 3 isolates i.e. $50 \%$ were sensitive to Imipenem and 2 isolates (i.e. $33.3 \%$ ) were sensitive to Imipenem. Isolates were resistant to Ampicillin, Cloxacillin, Cefazolin. Out of total of 4 isolates (1.37\%) of E. coli, 3 isolates (i.e. 75\%) were sensitive to Imipenem. Isolates were resistant to Ampicillin, Lomefloxacin and Cloxacillin. Proteus spp. are becoming difficult because of the development of resistance to newer antibiotics. So, inadvertent use of antibiotics must be discouraged.

\section{CONCLUSION}

In spite of use of effective antimicrobial agents, the problem of control of infection in burn patients still persists. Pseudomonas aeruginosa is still the major pathogen responsible for bacteraemia in burn wound infection. Other important bacteria are Staphylococcus aureus and Proteus spp. Pseudomonas aeruginosa is sensitive to Imipenem and ciprofloxacin; Proteus spp. to Ceftriaxone; Staphylococcus aureus to cloxacillin; E. coli to ciprofloxacin and Cefotaxime; Klebsiella spp. to ciprofloxacin and gentamicin; Streptococcus pyogenes to cefotaxime and cefoperazone; and Staphylococcus epidermidis to cloxacillin.

\section{KEYWORDS}

Burn Wound, Blood Culture, Antibiotic Sensitivity Pattern.

HOW TO CITE THIS ARTICLE: Kamlesh R, Ajay K, Sanjay N, et al. Bacteriological profile and antibiotic susceptibility pattern of blood culture isolates from burn patients. J. Evolution Med. Dent. Sci. 2017;6(83):5811-5814, DOI: 10.14260/jemds/2017/1261

\section{BACKGROUND}

Burn patients are ideal hosts for opportunistic infections (Cochran et al, 2002). The burn site remains relatively sterile during the first 24 hours; thereafter, colonisation of the wound by Gram-negative bacteria is common (Pruitt et al, 1998). Pseudomonas aeruginosa has emerged as a predominant member of the burn wound flora and in the

'Financial or Other Competing Interest': None.

Submission 09-09-2017, Peer Review 01-10-2017,

Acceptance 09-10-2017, Published 16-10-2017.

Corresponding Author:

Dr. Ajay Kumar,

Senior Resident,

Department of Microbiology,

Indira Gandhi Institute of Medical Sciences

(IGIMS), Patna-14.

E-mail:dr.ajay876@gmail.com

DOI: $10.14260 /$ jemds $/ 2017 / 1261$

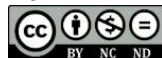

absence of topical therapy is cultured from the burn injuries of $70 \%$ patients by the third week (Church et al, 2006). Microorganisms routinely isolated from burn wounds include aerobic organisms like Staphylococcus aureus, Streptococcus pyogenes, E. coli, Klebsiella spp., Proteus, etc., anaerobic organisms like Bacteroides fragilis, Peptostreptococcus, Propionibacterium spp., Fusobacterium spp. and fungi like Aspergillus niger, Candida spp. and Zygomycetes (Revathi et al, 1998). Infection in the burn patient is a leading cause of morbidity and mortality and remains one of the most challenging concerns for doctors. ${ }^{1}$ The importance of preventing infection has been recognised in organised burn care since its inception and has followed recurring themes through the years. In spite of use of effective antimicrobial agents, the problem of control of infection in burn cases has not been entirely solved. ${ }^{2}$ The measures taken for infection control in burn patients are strict aseptic technique, use of 
sterile gloves and dressing materials, wearing masks for dressing changes and special separation of patients, either using private rooms or cubicles. ${ }^{3}$ There are three conditions which lead to the development of infection in burn patients: source of organisms, mode of transmission, and the susceptibility of the patient. Exogenous organisms from the hospital environment are generally more resistant to antimicrobial agents than endogenous organisms. ${ }^{4}$ All burn wounds become colonised by 72 hours after injury with the patient's own flora or with endemic organisms from the treatment facility. Bacteria colonise the surface of the wound and may penetrate the vascular eschar. Even with firm clinical evidence of sepsis, a definitive microbiological diagnosis of infection can sometimes be difficult to obtain. ${ }^{5}$ Blood cultures have a relatively low yield in the "burn" population and other anatomic sites of potential infection (e.g. wounds, respiratory tract and urine). Organisms may also reach the damaged area from the surrounding skin, clothing and systemic routes. 6

When the burn wound starts to heal, granulation tissue appears, and when the surface is clearing up, microclimate changes and Gram-positive flora, predominantly Staphylococcus comes back. Previously, $\beta$-haemolytic Streptococci was identified as the most serious threat and almost all burn patients become infected with this organism at some stage of their stay in hospital, and they often proceeded to a rapidly fatal course. ${ }^{7}$ In recent years, MRSA has become a particularly significant problem in Indian hospitals. The Burn Unit is a particularly fertile environment for MRSA, because of open wounds, frequent dressing changes, requiring handling by multiple healthcare workers, use of intraluminal devices and the inherent immunocompromised state of burn patients. ${ }^{8}$ Ultimately, the Gramnegative organisms emerge as the major cause of death in thermally injured patients. Among these, Pseudomonas aeruginosa was the worst offender. ${ }^{9}$ Majority of the victims of domestic burn are women and children. Patients in the burn ward are prone to cross contamination by airborne spread of the bacteria. ${ }^{10}$ Microorganisms carrying burn wound infection must be identified earlier and their sensitivity pattern should be done cautiously by selecting the proper and adequate antibiotics.

\section{MATERIALS AND METHODS}

Blood samples were collected from acutely burnt patients, being treated in the Rajendra Surgical Ward of the Patna Medical College, Hospital, Patna. This descriptive study was conducted from July 2004 to August 2005. The burn patients and their attendants were explained about the nature of the test done and information collected from them, in a scheduled proforma - as 1. Serial No., 2. Registration No., 3. Name, 4. Age, 5. Sex, 6. Religion, 7. Occupation, 8. Type of burn, 9. Percent of burn, 10. Interval between burn and admission. A total of 116 blood samples were processed during the study period. Five mL blood sample was collected from each adult, $2-5 \mathrm{~mL}$ from each child and 0.5-2 $\mathrm{mL}$ from infant's aseptically using $70 \%$ alcohol and $2 \%$ tincture of iodine and inoculated immediately into $50 \mathrm{~mL}$ Brain-Heart
Infusion (BHI) Broth with $0.025 \%$ of sodium polyanethol sulfonate as anticoagulant. In paediatrics cases, 1-2 $\mathrm{mL}$ of blood was inoculated in 5-10 mL of BHI broth. Negative result was followed by examining the broth daily for the sign of bacterial growth (turbidity, haemolysis, clot formation) and by doing final subculture at the end of seventh day. Bottles that showed sign of growth were further processed by Gram stain, followed by subculture on Blood agar, MacConkey agar, Mannitol salt agar and examined after 18-24 hours of incubation. Bacterial isolates were identified by colony morphology, Gram staining, catalase test, coagulase test, oxidase test, methyl red/Voges-Proskauer test (MR-VP), Triple sugar iron agar test, citrate utilisation test, Urease test and Sulfur Indole Motility (SIM) test using standard procedure for bacterial identification. Antibiotic Sensitivity Test was performed on Muller-Hinton Agar, blood agar and MacConkey Agar plates by Kirby-Bauer disc diffusion methods.

\section{Statistical Analysis}

The data collected was entered in the Microsoft excel computer programming and checked for any inconsistency. The results are presented in proportion/percentage.

\section{RESULT}

One hundred sixteen blood samples were collected from acutely burnt patients, being treated in the Rajendra Surgical Ward of the Patna Medical College and Hospital, Patna. This descriptive study was conducted from July 2004 to August 2005. In the present series of study, it is observed that out of a total of 17 isolates (5.84\%) of Staphylococcus aureus, 14 isolates (i.e. $82.35 \%$ ) were sensitive to Cloxacillin. Antibiotics resistant to Staphylococcus aureus were Gentamicin, Amikacin, Cotrimoxazole, Lomefloxacin. Out of a total of 27 (i.e. 9.28\%) isolates of Pseudomonas aeruginosa, 11 isolates (i.e. $40.74 \%$ ) were sensitive to Gentamicin and 13 isolates (i.e. $48.15 \%$ ) were sensitive to Imipenem. Isolates were resistant to Cloxacillin, Ampicillin, Cefazolin and Cotrimoxazole. Out of a total of 7 isolates (i.e. $2.41 \%$ ) of Proteus spp.; 6 isolates (i.e. 85.71\%) were sensitive to Imipenem. Isolates were resistant to Cloxacillin, Cefazolin, Cefoperazone. Out of a total of 6 isolates (i.e. $2.06 \%$ ) of Klebsiella spp., 3 isolates i.e. $50 \%$ were sensitive to Imipenem and 2 isolates (i.e. 33.3\%) were sensitive to Imipenem. Isolates were resistant to Ampicillin, Cloxacillin, Cefazolin. Out of total of 4 isolates (1.37\%) of E. coli, 3 isolates (i.e. $75 \%)$ were sensitive to Imipenem. Isolates were resistant to Ampicillin, Lomefloxacin and Cloxacillin. Proteus spp. are becoming difficult because of the development of resistance to newer antibiotics. So, inadvertent use of antibiotics must be discouraged. Antibiotic sensitivity pattern are given in Table 4 in $100 \%$ sensitive and $100 \%$ resistant form of both Gram-positive and Gram-negative organism. We excluded those antibiotics that are in the form of intermediate. [Table $1,2,3,4,5]$. 


\begin{tabular}{|c|c|c|c|c|c|}
\hline $\begin{array}{c}\text { Samples } \\
\text { taken at }\end{array}$ & $\begin{array}{c}\text { Total no. } \\
\text { of samples } \\
\text { Taken }\end{array}$ & $\begin{array}{c}\text { No. of samples } \\
\text { Showing } \\
\text { Positive Blood } \\
\text { Culture }\end{array}$ & $\begin{array}{c}\text { Percentage of } \\
\text { Samples showing } \\
\text { Positive Blood } \\
\text { Culture }\end{array}$ & $\begin{array}{c}\text { No. of samples } \\
\text { Showing } \\
\text { Negative Blood } \\
\text { Culture }\end{array}$ & $\begin{array}{c}\text { Percentage of } \\
\text { samples showing } \\
\text { Negative Blood } \\
\text { Culture }\end{array}$ \\
\hline $\begin{array}{c}\text { Within first 24 hrs. of } \\
\text { admission }\end{array}$ & $\begin{array}{c}116 \\
(100 \%)\end{array}$ & 0 & 0 & 116 & 100 \\
\hline $\begin{array}{c}\text { At the end of } \\
\text { first week }\end{array}$ & $\begin{array}{c}98 \\
(100 \%)\end{array}$ & 51 & 52 & 47 & 48 \\
\hline $\begin{array}{c}\text { At the end of } \\
\text { second week }\end{array}$ & $\begin{array}{c}77 \\
(100 \%)\end{array}$ & 10 & 12.98 & 67 & 87 \\
\hline
\end{tabular}

Table 1 Showing the Total Number and Percentage of Positive and Negative Cases of Blood Culture of Samples taken within 24 hours of Admission, at the end of First Week and at the End of Second Week

\begin{tabular}{|c|c|c|c|}
\hline Organism Isolated & $\begin{array}{c}\text { No. of isolates in blood } \\
\text { culture of samples taken within } \\
\text { 24 hrs of admission }\end{array}$ & $\begin{array}{c}\text { No. of isolates in blood } \\
\text { culture of samples taken at } \\
\text { the end of first week }\end{array}$ & $\begin{array}{c}\text { No. of isolates in blood } \\
\text { culture of samples taken at } \\
\text { the end of second week. }\end{array}$ \\
\hline Pseudomonas aeruginosa & Nil & 25 & 2 \\
\hline Proteus spp. & Nil & 6 & 1 \\
\hline Staphylococcus Aureus & Nil & 13 & 4 \\
\hline E. coli & Nil & 2 & 2 \\
\hline Klebsiella spp. & Nil & 5 & 1 \\
\hline Total & Nil & $\mathbf{5 1}$ & 10 \\
\hline \multicolumn{2}{r}{} \\
Table 2 Showing Number of Isolates in Blood Culture of Samples taken Within \\
24 hrs., at the end of First Week and at the end of Second Week of Admission
\end{tabular}

\begin{tabular}{|c|c|c|c|}
\hline Organism Isolated & $\begin{array}{c}\text { Percentage of isolates in } \\
\text { blood culture of sample } \\
\text { taken within 24 hrs. }\end{array}$ & $\begin{array}{c}\text { Percentage of isolates in } \\
\text { blood culture of samples taken } \\
\text { at the end of first week } \\
\text { of admission (n = 98) }\end{array}$ & $\begin{array}{c}\text { Percentage of isolates in blood } \\
\text { culture of samples taken at the } \\
\text { end of second week (n=77) }\end{array}$ \\
\hline Pseudomonas aeruginosa & Nil & 25.58 & 2.59 \\
\hline Proteus. Spp. & Nil & 6.12 & 1.29 \\
\hline Staphylococcus aureus & Nil & 13.26 & 5.19 \\
\hline E. coli & Nil & 2.04 & 2.59 \\
\hline Klebsiella spp. & Nil & 5.10 & 1.29 \\
\hline \multicolumn{2}{|r|}{ Table 3 Showing Type and Percentage of Isolates in Blood Culture of Samples taken Within } \\
24 hrs. of Admission, and at the End of First Week, and at the end of Second Week of Admission \\
\hline
\end{tabular}

\begin{tabular}{|c|c|c|}
\hline Organism Isolated & $\begin{array}{l}\text { Total No. } \\
\text { of Isolates }\end{array}$ & Percentage \\
\hline Pseudomonas aeruginosa & 27 & 9.28 \\
\hline Proteus spp. & 7 & 2.41 \\
\hline Staphylococcus aureus & 17 & 5.84 \\
\hline E.coli & 4 & 1.37 \\
\hline Klebsiella spp. & 6 & 2.06 \\
\hline Total & 61 & 20.96 \\
\hline \multicolumn{3}{|c|}{$\begin{array}{c}\text { Table } 4 \text { Showing Frequency of Total Isolates of Blood } \\
\text { Culture, Recorded from Samples taken } \\
\text { Within } 24 \text { hrs. of Admission, at the end of First } \\
\text { Week and at the end of Second Week }\end{array}$} \\
\hline
\end{tabular}

Sensitivity Pattern of the Isolates in Blood Culture to different Antibiotics

In the present series of study, it is observed that out of a total of 17 isolates (5.84\%) of Staphylococcus aureus, 14 isolates (i.e. $82.35 \%$ ) were sensitive to Cloxacillin. Antibiotics resistant to Staphylococcus aureus were Gentamicin, Amikacin, Cotrimoxazole, Lomefloxacin,

Out of a total of 27 (i.e. 9.28\%) isolates of Pseudomonas aeruginosa, 11 isolates (i.e. $40.74 \%$ ) were sensitive to Gentamicin and 13 isolates (i.e. 48.15\%) were sensitive to Imipenem. Isolates were resistant to Cloxacillin, Ampicillin, Cefazolin and Cotrimoxazole.

Out of a total of 7 isolates (i.e. $2.41 \%$ ) of Proteus spp., 6 isolates (i.e. $85.71 \%$ ) were sensitive to Imipenem. Isolates were resistant to Cloxacillin, Cefazolin, Cefoperazone.
Out of a total of 6 isolates (i.e. 2.06\%) of Klebsiella spp., 3 isolates i.e. $50 \%$ were sensitive to Imipenem and 2 isolates (i.e. 33.3\%) were sensitive to Gentamicin. Isolates were resistant to Ampicillin, Cloxacillin, Cefazolin.

Out of a total of 4 isolates $(1.37 \%)$ of E. coli, 3 isolates (i.e $75 \%)$ were sensitive to Imipenem. Isolates were resistant to Ampicillin, Lomefloxacin and Cloxacillin.

\begin{tabular}{|c|c|c|}
\hline Organism Isolated & $\begin{array}{l}\text { Sensitive } \\
(100 \%)\end{array}$ & Resistant (100\%) \\
\hline $\begin{array}{l}\text { Pseudomonas } \\
\text { aeruginosa }\end{array}$ & $\begin{array}{c}\text { Gentamicin, } \\
\text { Imipenem }\end{array}$ & $\begin{array}{l}\text { Cloxacillin, Ampicillin, } \\
\text { Cotrimoxazole, Cefazolin }\end{array}$ \\
\hline Proteus spp. & Imipenem & $\begin{array}{l}\text { Cloxacillin, Cefazolin, } \\
\text { Cefoperazone }\end{array}$ \\
\hline $\begin{array}{l}\text { Staphylococcus } \\
\text { aureus }\end{array}$ & Cloxacillin & $\begin{array}{c}\text { Gentamicin, Amikacin, } \\
\text { Cotrimoxazole, } \\
\text { Lomefloxacin] }\end{array}$ \\
\hline Klebsiella spp. & \begin{tabular}{|l|} 
Imipenem, \\
Gentamicin
\end{tabular} & $\begin{array}{l}\text { Ampicillin, Cloxacillin, } \\
\text { Cefazolin }\end{array}$ \\
\hline E. coli & Imipenem & $\begin{array}{l}\text { Ampicillin, Cloxacillin, } \\
\text { Lomefloxacin }\end{array}$ \\
\hline \multicolumn{3}{|c|}{$\begin{array}{c}\text { Table } 5 \text { Showing Antibiotic Sensitivity Pattern } \\
\text { of Isolates in Blood Culture to Different Antibiotics }\end{array}$} \\
\hline
\end{tabular}

\section{DISCUSSION}

Samples of blood taken within 24 hours of admission shows no growth of bacteria because the organism did not pass into 
the blood, although the wound got heavily contaminated. Around one week after burn injury, the organism started entering the blood from the surfaces causing bacteraemia and septicaemia. In the present series, it has been observed that Pseudomonas aeruginosa is mostly sensitive to Amikacin and Imipenem. $38.46 \%$ isolates are sensitive to Amikacin and $42.3 \%$ isolates are sensitive to Imipenem. $75.5 \%$ isolates of Staphylococcus aureus are sensitive to Cloxacillin. 98\% isolates of Proteus spp. are sensitive to Imipenem. 44.4\% isolates of E. coli are sensitive to Ciprofloxacin and 55.5\% isolates are sensitive to Imipenem. 50\% isolates of Klebsiella spp. are sensitive to Imipenem and $50 \%$ isolates to Gentamicin. $75 \%$ isolates of Streptococcus pyogenes are sensitive to Cefotaxime and $12.5 \%$ are sensitive to Cefoperazone. $83.3 \%$ isolates are sensitive to Cloxacillin and $8.3 \%$ isolates are sensitive to Cefazolin. It has also been observed that most of the isolates show resistance to some antibiotics. In this study, it has been seen that Pseudomonas aeruginosa is resistant to Cloxacillin, Lomefloxacin, Cotrimoxazole, Ampicillin and Cotrimoxazole. Staphylococcus aureus is resistant to Gentamicin, Cotrimoxazole, and Lomefloxacin. E. coli is resistant to Amikacin, Cloxacillin, Lomefloxacin, Cefotaxime and Cotrimoxazole. Klebsiella spp. Is resistant to Ampicillin, Cloxacillin, Ciprofloxacin and Lomefloxacin. Streptococcus pyogenes is resistant to Cotrimoxazole, Ciprofloxacin, Amikacin, Gentamicin and Staphylococcus epidermidis is resistant to Ceftriaxone, Cotrimoxazole, Ampicillin, Amikacin and Gentamicin. This increasing tendency to bacterial resistance is because of inadvertent use of antibiotics in burn cases. Here lies the importance of bacteriological isolation and its proper antibiotic selection to avoid use of unnecessary antibiotic administration to the patient. Above facts are also accepted by Joan Weber, R.N., Albert McManus, 1990. They stated that systemic antimicrobial treatment must be thoughtfully considered in the care of burn patients to prevent the emergence of resistant organisms. Organism isolated in blood, culture also shows the same sensitivity pattern as that of isolates of wound swab culture. The above findings are also shown by Jackson, Lowbury \& Topley, 1951, who stated that incidence of Pseudomonas infection in burns is rising and is likely to cause fatal septicaemia. Blach, 1965, stated that septicaemia is more common in severely burnt patients and microorganisms isolated from the blood stream are usually found in the burn wound.

\section{CONCLUSION}

In spite of use of effective antimicrobial agents, the problem of control of infection in burn patients still persists. In cases of burn wounds, it is important to do an immediate infection workup and administer the appropriate antibiotics. Patients in the burn ward are more prone to cross contamination by airborne spread of the bacteria. Beddings, pillows, blankets and mattresses are proved to be the reservoir of microorganisms. Thermal burn is the most common type of burn. Next is the scald burn which is more common among children. Young females, mostly those involved in domestic works are the major victims of burn injury. Most common isolates from upper extremity are Gram-positive cocci, whereas most common isolates from lower extremity, back, perineum, buttocks, and thigh are Gram-negative bacilli. Pseudomonas aeruginosa is still the major pathogen responsible for bacteraemia in burn wound infection. Other important bacteria are Staph. aureus and Proteus spp. Specific isolates of wound swab culture and blood cultures are sensitive to same antibiotics. Pseudomonas aeruginosa is sensitive to Imipenem and ciprofloxacin; Proteus spp. to Ceftriaxone; Staphylococcus aureus to cloxacillin; E. coli to ciprofloxacin and Cefotaxime; Klebsiella spp. to ciprofloxacin and gentamicin; Streptococcus pyogenes to cefotaxime and cefoperazone; and Staphylococcus epidermidis to cloxacillin. Staphylococcus aureus and Proteus spp. is becoming difficult because of the development of resistance to newer antibiotics. So, inadvertent use of antibiotics must be discouraged. On correlating the burn wound swab culture and blood culture, it is seen that most of the isolates of blood culture and their corresponding wound swab culture are identical. If antimicrobial therapy is indicated to treat specific infection, it should be tailored to the specific susceptibility pattern of the organisms, as soon as this information is available. Most of the cases of late arrival in hospital shows growth of microorganisms in wound swab culture.

\section{REFERENCES}

[1] Alborzi A, Pourabbas BA, Salehi H, et al. Prevalence and pattern of antibiotic sensitivity of methicillinsensitive and methicillin-resistant staphylococcus aureus in Shiraz-Iran. Iranian J Med Sciences 2000;25(1-2):1-8.

[2] Barillo DJ, Brigham PA, Kayden DA, et al. Burn Blood infection: the fire safe. A burn Prevention 1998;22:134-45.

[3] Bowler PG. Wound pathophysiology, infection and therapeutic options. Annals of Medicine 2002;34(6):419-27.

[4] David N, Herndon A. Research program report in Shriners burn hospital Galveston, Texas, 2004.

[5] Herndon DN, Spies M. Modern burn care. Semin Pediatr Surg 2001;10(1):28-31.

[6] Alekseev AA, Iakovlev VP, Fedorov VD. Infection in burn patients: the problems of pathogenesis, prevention and treatment. Khirurgiia (Mosk) 1999;(6):4-9.

[7] Kauvar DS, Acheson E, Reeder J, et al. Comparison of battlefield-expedient topical antimicrobial agents for the prevention of burn wound sepsis in a rat model. Journal of Burn Care and Rehabilitation 2005;26(4):357-61.

[8] Keen A, Knoblock L, Edelman L, et al. Effective limitation of blood culture use in the burn unit. J Burn Care \& Rehabil 2002;23(3):183-9.

[9] Mayhall CG. The epidemiology of burn wound infections: then and now. Clin Infect Dis 2003;37(4):543-50.

[10] Kehinde AO, Ademola SA, Okesola AO, et al. Pattern of bacterial pathogens in burn wound infections in Ibadan, Nigeria. Annals of Burns and Fire Disasters 2004;17:12-15. 